\title{
Anatomic considerations for central venous cannulation
}

This article was published in the following Dove Press journal:

Risk Management and Healthcare Policy

12 April 201 I

Number of times this article has been viewed

\author{
Michael P Bannon \\ Stephanie F Heller \\ Mariela Rivera \\ Department of Surgery, \\ Division of Trauma, Critical Care, \\ and General Surgery, Mayo Clinic, \\ Rochester, Minnesota, USA
}

Correspondence: Michael P Bannon Mayo Clinic, Department of Surgery, 200 First Street SW, Rochester, MN 55905, USA

Tel +I 5072556365

Fax + I 5072559872

Email bannon.michael@mayo.edu

\begin{abstract}
Central venous cannulation is a commonly performed procedure which facilitates resuscitation, nutritional support, and long-term vascular access. Mechanical complications most often occur during insertion and are intimately related to the anatomic relationship of the central veins. Working knowledge of surface and deep anatomy minimizes complications. Use of surface anatomic landmarks to orient the deep course of cannulating needle tracts appropriately comprises the crux of complication avoidance. The authors describe use of surface landmarks to facilitate safe placement of internal jugular, subclavian, and femoral venous catheters. The role of real-time sonography as a safety-enhancing adjunct is reviewed.
\end{abstract}

Keywords: Internal jugular vein, cannulation, ultrasound, venipuncture

\section{Introduction}

Percutaneous techniques revolutionized vascular cannulation. They essentially eliminated the need for open cutdown procedures and the associated wound-related morbidity, but percutaneous techniques left the operating physician exclusively reliant upon the relationships between surface anatomic landmarks and the underlying deep anatomic structures. Clinicians now insert more than five million percutaneous central venous catheters annually, with an overall complication rate of $15 \% .{ }^{1}$ These complications include infection, thrombosis, occlusion, and, in particular, mechanical complications which usually occur during insertion and are intimately related to the anatomic relationships of the central veins.

Working knowledge of surface and deep anatomy minimizes these latter complications. Use of surface anatomic landmarks to orient the deep course of cannulating needle tracts appropriately comprises the crux of complication avoidance. Mind's eye visualization of relevant deep anatomic relationships ${ }^{2-4}$ not only increases the safety and efficiency of the procedure but also enhances the operator's ability to troubleshoot difficult cannulations. Percutaneous approaches should never be a foray into a "black box" of uncharted territory, but should always be performed into a wellvisualized mental map of the underlying structures. Real-time ultrasound visualization now enhances the safety of internal jugular and femoral venous cannulations. Ultrasound also offers the axillary vein as a "visible" alternative to blind subclavian approaches.

\section{General considerations Preparation, draping, and sterile technique}

Central venous cannulation must be performed with scrupulous attention to antisepsis and sterile technique to prevent catheter-related infection, a complication associated 
with significant morbidity and preventable mortality. Chlorhexidine, the most efficacious antiseptic, ${ }^{5,6}$ should be painted over the entirety of exposed cutaneous surfaces. Because surface anatomic landmarks are essential guides to the relevant deep anatomic structures, they must remain clearly exposed after preparation and draping. Outside the prepared procedural field, the patient should be draped from head to toe. The operator should don hat, mask, gown, and gloves. These techniques have been shown to decrease catheter-related infection. ${ }^{7}$

\section{Patient positioning}

Unless contraindicated by emergent circumstances, the patient should be placed in the Trendelenburg position for internal jugular and subclavian cannulation. Often, the indication for central venous line placement is volume resuscitation, and veins are correspondingly poorly distended at the time of placement. Trendelenburg position allows gravity to enhance central venous filling to create a larger target for venipuncture and minimize risk of air embolus. However, even adequately filled veins are at risk of collapse during gentle puncture of the front wall with a needle. Collapse increases the risk of back wall puncture, ${ }^{8}$ with potential complications of hematoma, hemorrhage, and/or extravenous placement of a guidewire, dilator, or large-bore catheter. Especially ominous, collapse is associated with potential intra-arterial placement of these devices. Vein collapse with transluminal puncture is much less likely with subclavian than internal jugular venipuncture, because the subclavian vein is suspended within soft tissue underlying the clavicle. For femoral venipuncture, it is generally recognized that the reverse Trendelenburg position will not substantially increase the caliber of the vein. Furthermore, reverse Trendelenburg position may be contraindicated by hemodynamic instability accompanying volume depletion. As discussed below, positional maneuvers affecting the head and shoulders can affect the caliber and anatomic relationship of the veins in relation to local structures during cannulation.

\section{Seldinger technique}

The Seldinger technique is a general means of placing a catheter into an artery or vein. The vessel is accessed with a relatively narrow bore needle through which a guidewire is advanced; the needle is removed, and the catheter is then inserted over the guidewire. This technique was introduced in the early 1950s as an innovation for arteriography and offered advantages over previous needle-alone and catheter-through-the-needle techniques. ${ }^{9}$
Despite the advantage of allowing a catheter to enter a vessel through an opening no larger than the catheter itself, the Seldinger technique did not completely replace the catheter-through-the-needle technique in central venous cannulation until the early 1980s.

\section{Catheter tip position}

Ideally the catheter tip lies at the junction of the superior vena cava and the right atrium. This location ensures a proximal position with high blood flow which prevents thrombosis (especially of concern with parenteral nutrition solutions and chemotherapeutic agents) and yet lies outside the atrium and thus prevents arrhythmias from catheter tip irritation of the right atrial wall. The surface landmark corresponding to this position is the angle of Louis, ie, the junction between the manubrium and the sternum. Measurement of the distance between the cutaneous puncture site and the angle of Louis along the trajectory of the vein allows the operator to estimate the appropriate length of catheter for insertion. Pikwer et al found catheter tip malposition, defined as extrathoracic or ventricular locations, in $3.3 \%$ of insertions; the highest incidence occurring from the right subclavian approach $(9.1 \%)$ and the lowest from the right internal jugular approach $(1.4 \%) .{ }^{10}$ The present authors consider distal intrathoracic and atrial locations to also represent malpositions, and thus the data of Pikwer et al may under-represent the incidence. An appropriate radiographic landmark to guide appropriate catheter tip position on chest $\mathrm{x}$-ray is the right tracheobronchial angle, which is always inferior to the cephalad origin of the superior vena cava by a median distance of $1.5 \mathrm{~cm}$; this landmark is always at least $2.9 \mathrm{~cm}$ superior to the atriocaval junction. ${ }^{11}$ Thus, catheter tips positioned approximately $3 \mathrm{~cm}$ below the right tracheobronchial angle will lie in close proximity to the atriocaval junction but will remain extracardiac in location (Figure 1). Fluoroscopically-assisted measurements of guidewire length from the cutaneous puncture site to the superior vena cava-atrial junction have revealed mean distances of $16.0 \mathrm{~cm}$ for the right internal jugular, $18.4 \mathrm{~cm}$ for the right subclavian, $19.1 \mathrm{~cm}$ for the left internal jugular, and $21.2 \mathrm{~cm}$ for the left subclavian veins, respectively. ${ }^{12}$ These measurements are useful guides, but long-term catheters should be positioned precisely under fluoroscopy, and the position of short-term lines should be confirmed with a postprocedure chest x-ray.

\section{Dilators and introducer sheaths}

Large caliber catheters, such as dialysis catheters, are placed after the passage of a stiff dilator creates a 


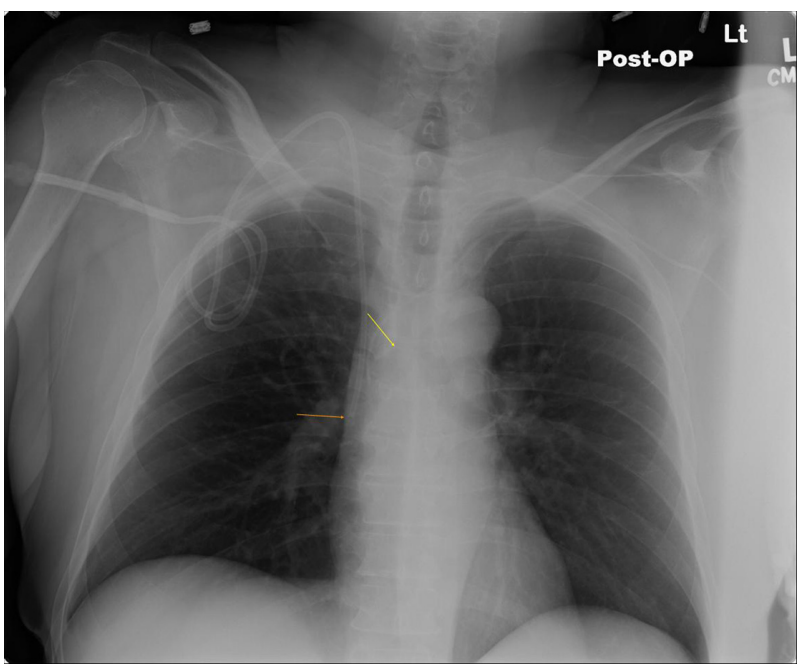

Figure I Long-term right internal jugular venous catheter (tip at orange arrow) appropriately positioned relative to right tracheobronchial angle (at yellow arrow).

subcutaneous tract. Soft silastic catheters for long-term use are too flimsy to be placed over a guidewire; they are placed through a peel-away introducer sheath which is itself placed while loaded onto an obturating dilator. These dilators, being wide-bore and stiff, cause serious injury to any structure unintentionally punctured. Vascular injury may lead to life-threatening hemorrhage or cardiac tamponade. ${ }^{13}$ The danger of vessel injury makes an understanding of the underlying vein trajectory critical. From the right internal jugular and left subclavian approaches, the veins respectively take straight and gently curving trajectories to the superior vena cava (Figure 2). However, the right subclavian vein takes a near-right angle turn into

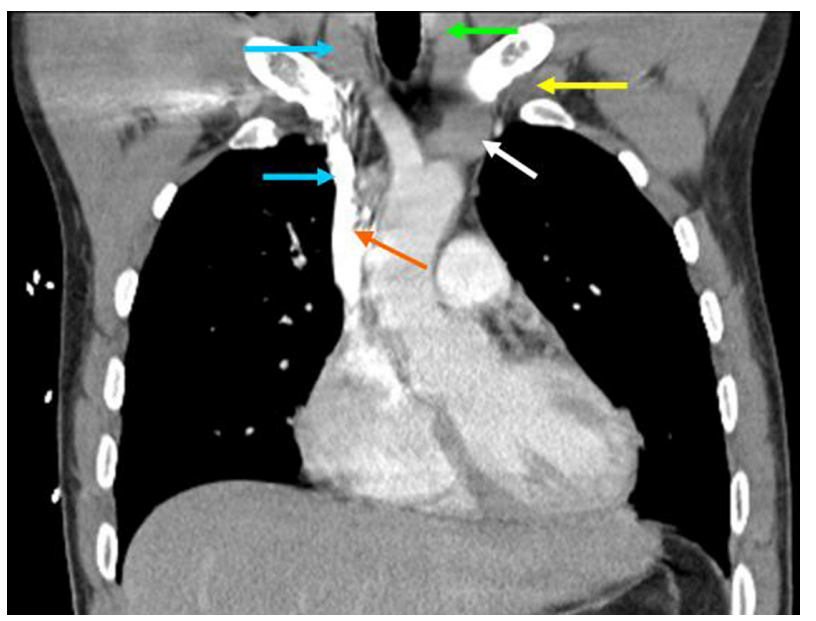

Figure 2 Computed tomography scan showing venous course from right internal jugular vein (blue arrows) and venous course from left subclavian vein (yellow arrow) through the innominate vein (white arrow) to superior vena cava (orange arrow); course from left internal jugular vein (green arrow) with turns at junctions with innominate vein and superior vena cava.

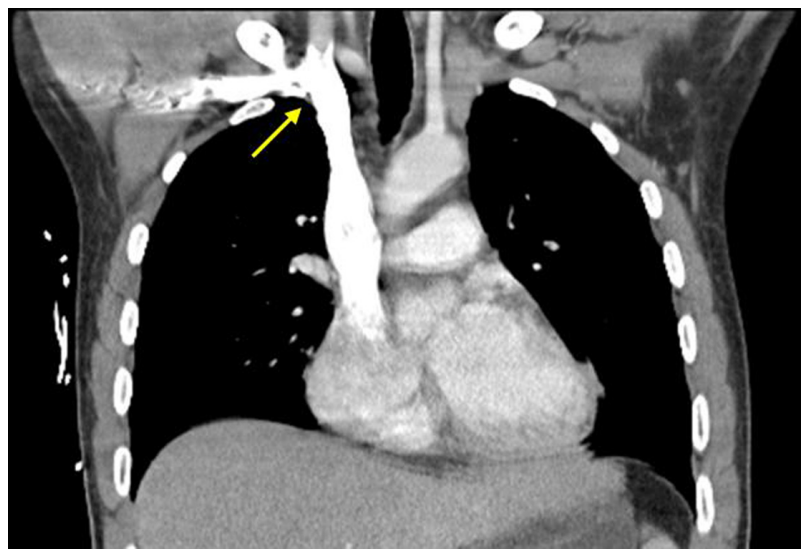

Figure 3 Computed tomography scan showing right angle junction (arrow) of right subclavian vein with superior vena cava.

the superior vena cava (Figure 3), and the left internal jugular approach incorporates two turns, one into the brachiocephalic vein and a second into the superior vena cava (Figure 2). These turns create potential for the venous side walls to be punctured by a dilator failing to negotiate a curve appropriately.

A dilator should never be given the opportunity to cause such venous injury. Dilators are used to develop a subcutaneous tract. They should be advanced to the level of the adventitial vein surface but never more than millimeters into the vein itself. The operator will know the depth at which the vein was accessed during venipuncture, and this depth should be the maximal distance the dilator is inserted. Advancing the full length of the dilator through the subcutaneous tract and into the vein risks venous injury. When placing catheters through peel-away sheaths, the sheath and dilator are advanced together to the level of the vein wall and then the sheath is advanced off from the stationary dilator. The sheath, but not the dilator, is advanced into the vein lumen. The right internal jugular vein and the left subclavian vein are the preferred sites for cannulation with catheters requiring introducer sheaths to avoid kinking of the sheath at the turns associated with the right subclavian and left internal jugular approaches. The right internal jugular and left subclavian veins are also the preferred approaches for wide-bore stiff dialysis catheters that carry a greater risk of venous injury in the alternative positions for the same anatomic reasons. If the right subclavian vein or left internal jugular vein are selected due to other anatomic considerations (such as thrombosis or stenosis of the preferred veins), large caliber lines and introducer sheaths should be placed with fluoroscopic guidance so that the operator may ensure the expected venous trajectory is followed during insertion; fluoroscopy 
also allows kinking of introducer sheaths to be recognized immediately.

\section{Ultrasound visualization}

Relative advantages of real-time ultrasonographic localization are discussed below for each vessel. Given the superficial location of the central veins at the sites of venipuncture, a high frequency probe of $7.5 \mathrm{mHz}$ creates optimal images. Ultrasound equipment can be easily used within a sterile field (Figure 4). Ultrasound does not obviate the need for anatomic knowledge, so surface anatomic landmarks remain necessary for orientation of both cannulating needles and the ultrasound probe itself. However, ultrasound affords the operator an "inside view" of vascular anatomy and eliminates the total reliance on mind's eye visualization of deep structures that was originally an inherent part of all percutaneous techniques. Real-time sonography provides a means to aim the cannulating needle directly toward the internal jugular, axillary, and femoral veins while avoiding puncture of the accompanying arteries. Needle guides which orient the needle within the field of view along the path of the ultrasound beam can facilitate venipuncture. Although subclavian cannulation remains a blind technique, the use of ultrasound has improved outcomes for internal jugular vein cannulation, and is considered a current standard of care for cannulation at this site..$^{14,15}$

\section{Internal jugular cannulation}

The internal jugular vein is often the access site of choice for central venous cannulation. Advantages include a superficial location, easy ultrasonic visualization, and a straight course to the superior vena cava (on the right). Internal jugular

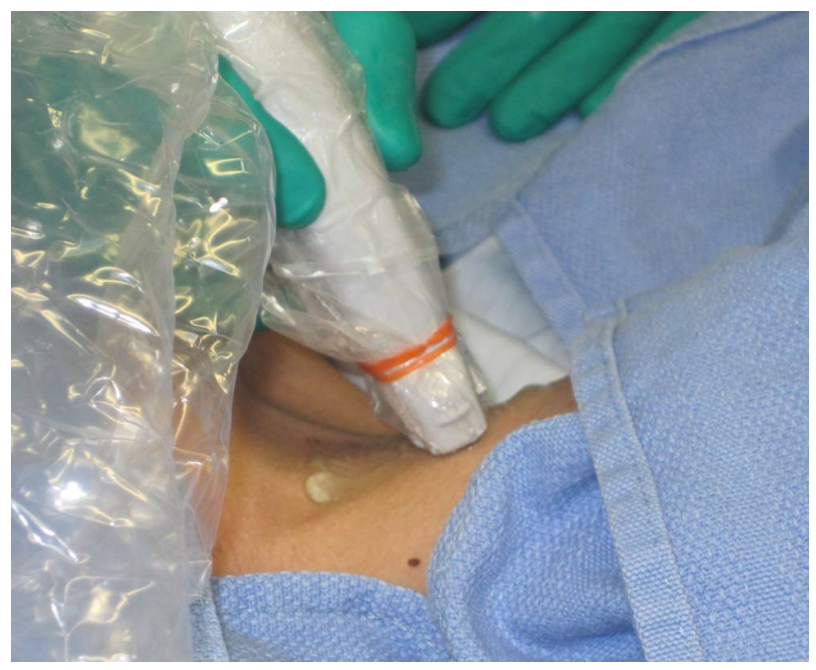

Figure 4 Ultrasound probe prepared within sterile sheath. cannulation avoids the subclavian "pinch-off syndrome". Furthermore, for renal failure patients, it avoids potential subclavian vein stenosis which would preclude use of the extremity for hemodialysis access via arteriovenous shunt/fistula.

\section{Surface anatomy and venipuncture technique}

There are three percutaneous approaches to the internal jugular vein, ie, anterior, central, and posterior. The authors' preferred approach for internal jugular cannulation is the central approach. The essential surface anatomy is comprised of the borders of Sedillot's triangle, the sternal head of the sternocleidomastoid muscle medially, the clavicular head of the sternocleidomastoid laterally, and the superior border of the medial third of the clavicle inferiorly (Figure 5). For the awake supine patient, the borders of this triangle are accentuated by active head-raising. For the noncooperative or obese patient, the triangle is best defined by initial palpation of the trachea and then drawing the fingers laterally over the sternal head of the sternocleidomastoid into the depression of the triangle. Cannulation begins with cutaneous puncture at the superior apex of this triangle. The needle and syringe are angled $45^{\circ}$ off a coronal plane, and the needle is advanced in a sagittal plane.

The internal jugular vein lies immediately posterior to the apex of Sedillot's triangle with a frequency of $97 \%$ on the right and $79 \%$ on the left, ${ }^{16}$ and is thus relatively superficial in location. The internal jugular vein will be accessed at a needle depth of $1.0-1.5 \mathrm{~cm}$ beneath the cutaneous surface ${ }^{8}$ in most patients. If the vein is not accessed at this superficial level, one should consider that the advancing needle

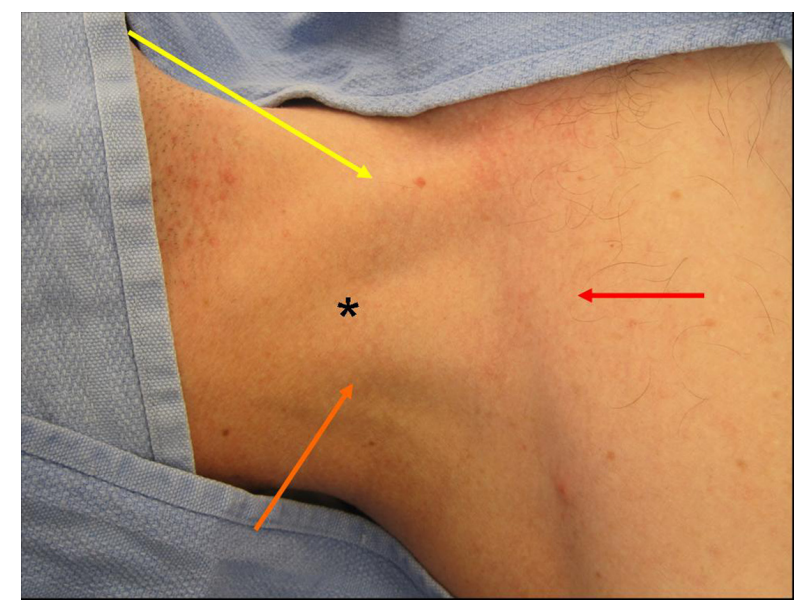

Figure 5 Sedillot's triangle: sternal head of sternocleidomastoid (yellow arrow), clavicular head sternocleidomastoid (orange arrow), clavicle (red arrow); and position of appropriate cutaneous puncture at apex of triangle (black star). 
may have opposed the anterior and posterior walls of the internal jugular vein, collapsing the lumen, and allowing transluminal passage of the needle out of the vein through its posterior wall. ${ }^{17}$ "Back-walling" the internal jugular vein in this manner risks injury to deeper structures, in particular the pleura and lung. Consequently, Cahill recommends slowly withdrawing the needle if it has been advanced $2.0-2.5 \mathrm{~cm}$ below the cutaneous surface without successful cannulation. ${ }^{18}$ Slow withdrawal eliminates the forward pressure collapsing the vein, allows luminal patency to be re-established, and will likely result in a reassuring blood return into the needle. If this maneuver is unsuccessful, the needle should be advanced from the cutaneous puncture site with a slightly lateral trajectory to puncture the vein posterior to the clavicular head of the sternocleidomastoid. The immediate goal is to access the internal jugular vein and avoid puncture of the pulmonary apex and carotid artery.

To initiate the posterior approach, the needle is placed at the posterior border of the sternocleidomastoid muscle, at a point one-third of the way from the sternoclavicular joint to the mastoid process. The external jugular vein crosses the sternocleidomastoid muscle at this point and, when visible, serves as a useful landmark (Figure 6). The needle

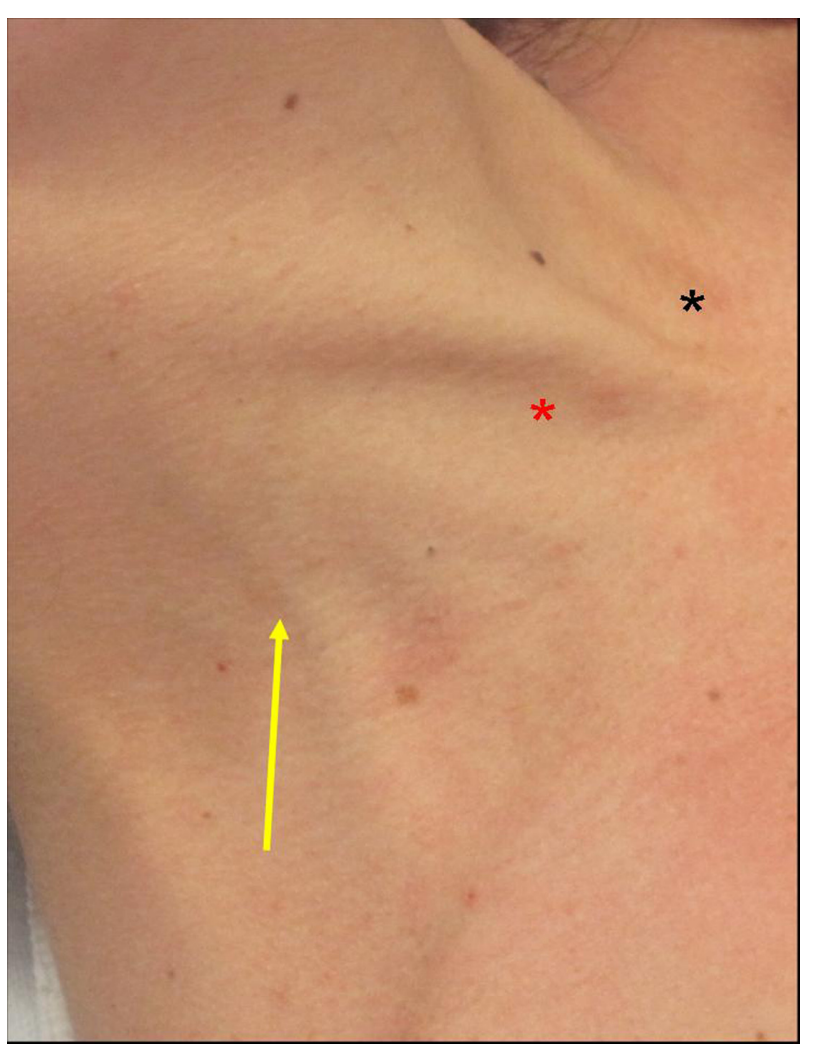

Figure 6 External jugular vein crossing the posterior border of sternocleidomastoid (arrow), Sedillot's triangle (red star), and sternal notch (black star). is advanced toward the ipsilateral sternoclavicular joint at an angle of $30^{\circ}-40^{\circ}$ off the skin. ${ }^{16,19}$ The cutaneous puncture site for the anterior approach lies at the medial border of the sternocleidomastoid at the level of the cricoid cartilage. The needle tip should pass medial to the sternocleidomastoid directed $30^{\circ}-45^{\circ}$ posteriorly from a coronal plane and $15^{\circ}-45^{\circ}$ laterally from a sagittal plane.

\section{Deep anatomic considerations \\ Course}

It is important to recognize that the deep courses of the left and right internal jugular veins are not bilaterally symmetric. The right internal jugular vein follows a direct course inferiorly to the superior vena cava (Figure 2). The left internal jugular vein courses to the right after it joins the left subclavian vein to become the innominate vein, and the innominate vein subsequently courses inferiorly as it joins the superior vena cava (Figure 2). The angulation at the junction of the left internal jugular vein and innominate vein can create difficulties when passing catheters through introducer sheaths. First, the introducer sheath may become kinked as it negotiates this angle, preventing the passage of an intravascular catheter through the sheath. In this event, seen clinically during the passage of soft, long-term tunneled catheters through "peel-away" introducer sheaths, the introducer sheath must be partially withdrawn so that it will straighten and allow passage of the catheter; if the sheath becomes permanently crimped and narrowed, it must be exchanged for a new sheath inserted to a shallower intravenous depth. Alternatively, the tip of the introducer sheath may lie so close to the inferior wall of the innominate vein that the catheter kinks as it exits the introducer sheath. This latter situation will cause damping of pressures monitored through pulmonary artery or central venous pressure catheters, which can be rectified by partial withdrawal of the introducer. Finally, a stiff dilator inserted to a maximal depth has the potential to puncture the inferior wall of the innominate vein at the jugular-innominate junction. To prevent such complications, dilators should be inserted only far enough to dilate the subcutaneous tract as discussed earlier.

Given the more consistent presence of the right internal jugular vein immediately deep to the apex of Sedillot's triangle, ${ }^{16}$ and the nonlinear course from the left internal jugular vein to the superior vena cava, right internal jugular vein cannulation is preferred whenever feasible. Furthermore, total complications have been found more frequently after left versus right internal jugular cannulation. ${ }^{20}$ 


\section{Relationship to carotid artery}

The occurrence of carotid arterial puncture is due to the close relationship between the internal jugular vein and the carotid artery. Understanding this relationship is crucial for avoidance of this complication. The internal jugular vein generally lies anterolateral to the carotid artery. However, the vein may also lie directly anterior to the artery, predisposing to arterial puncture. Chandrasekaran found this relationship in $26 \%$ on the right and $20 \%$ on the left in young healthy volunteers. ${ }^{20}$ Using clock-dial positions to describe the position of the vein relative to the artery in the central position, Turba et al found the left internal jugular vein to lie at the expected anterolateral 10 o'clock position in $75 \%$ of subjects, at the direct lateral position of 9 o'clock in only $9 \%$, and at the more anterior positions of 11 o'clock and 12 o'clock with a combined incidence of $15 \% .^{21}$ (The clock-dial positions are described from the perspective of the operator standing at the patient's head and facing the patient's feet). The right internal jugular vein was found more commonly in the 12 o'clock anterior and 1 o'clock positions in $20 \%$ of subjects, in the anterolateral 2 o'clock position in $71 \%$, and similarly in the directly lateral 3 o'clock position in $9 \%{ }^{22}$ Very rarely, the internal jugular vein lies medial to the carotid artery. The anterior positions of the vein predispose to arterial puncture through the mechanism of transluminal through-and-through venous puncture as already described.

\section{Alternative positioning and adjunctive maneuvers}

The effects of head rotation, carotid artery palpation, and Trendelenburg position on internal jugular vein diameter have been studied. Contralateral rotation of the head brings the sternocleidomastoid muscle into an anterior position over the internal jugular vein impeding cannulation, ${ }^{23}$ especially from the anterior approach. ${ }^{24}$ Contralateral rotation beyond $40^{\circ}$ increases the anterior-posterior overlap of the internal jugular vein with the carotid artery, increasing the risk of arterial puncture. ${ }^{25}$ Carotid artery palpation diminishes internal jugular vein diameter. ${ }^{24}$ Trendelenburg position reliably increases internal jugular vein diameter. ${ }^{24,26,27}$ Bazaral and Harlan ${ }^{23}$ found $14^{\circ}$ of Trendelenburg position to increase internal jugular vein cross-sectional area imaged by ultrasound significantly, from $0.78 \mathrm{~cm}^{2}$ to $1.24 \mathrm{~cm}^{2}$. Positive end-expiratory pressure also increases internal jugular vein diameter, but to a lesser degree than the Trendelenburg position. ${ }^{27}$ Consequently, Trendelenburg position should be employed whenever feasible, the carotid artery should not be palpated during venipuncture, and the head should be rotated to the contralateral side only so far as to provide access to the neck.

\section{Anatomic complications}

The primary complications are puncture/cannulation of the internal carotid artery and pneumothorax. Puncture of the pulmonary apex with attendant pneumothorax is prevented by avoiding passage of the needle beyond a depth of $1-2 \mathrm{~cm}$. Cahill emphasizes the superficial depth of the internal jugular vein beneath Sedillot's triangle by totaling the expected thicknesses of the structures overlying the internal jugular vein, ie, $2 \mathrm{~mm}$ for skin, 3-6 $\mathrm{mm}$ for superficial fascia and platysma, and 5-6 mm for the sternocleidomastoid. ${ }^{18}$

The carotid artery generally lies medial to the internal jugular vein, and carotid artery puncture is prevented by avoiding medial angulation of the needle as it passes below the cutaneous surface. At times, the carotid artery lies posterior to the internal jugular vein and is accessed after the cannulating needle has passed through-and-through the internal jugular vein.

Additionally, carotid puncture is also avoided by staying high in Sedillot's triangle. As one progresses lower in the triangle, the carotid moves anteromedially in relation to the internal jugular vein, taking a "side-by-side" position, while the internal jugular vein moves deeper, requiring deeper placement of the tip of the needle for cannulation. This makes puncture of the carotid without first passing through the internal jugular vein more likely.

\section{Role of ultrasound}

The authors routinely use real-time ultrasound guidance for internal jugular vein cannulation. The issues created by the close proximity of the carotid artery to the internal jugular vein are most efficaciously dealt with by ultrasound localization of the carotid artery and internal jugular vein in real time during venipuncture. Ultrasonographic imaging visually differentiates the internal jugular vein and common carotid artery, facilitates venipuncture rather than arterial puncture, guards against through-and-through puncture of the internal jugular vein, and prevents deep passage of a needle into deep cervical and thoracic structures.

Ultrasound imaging through Sedillot's triangle will demonstrate the carotid artery and internal jugular vein as two sonolucent circles. The artery is recognized as the smaller diameter, noncompressible vessel with a visible pulsation (Figure 7).

Cervical vessel diameters have been measured by ultrasound for a variety of clinical correlations. 


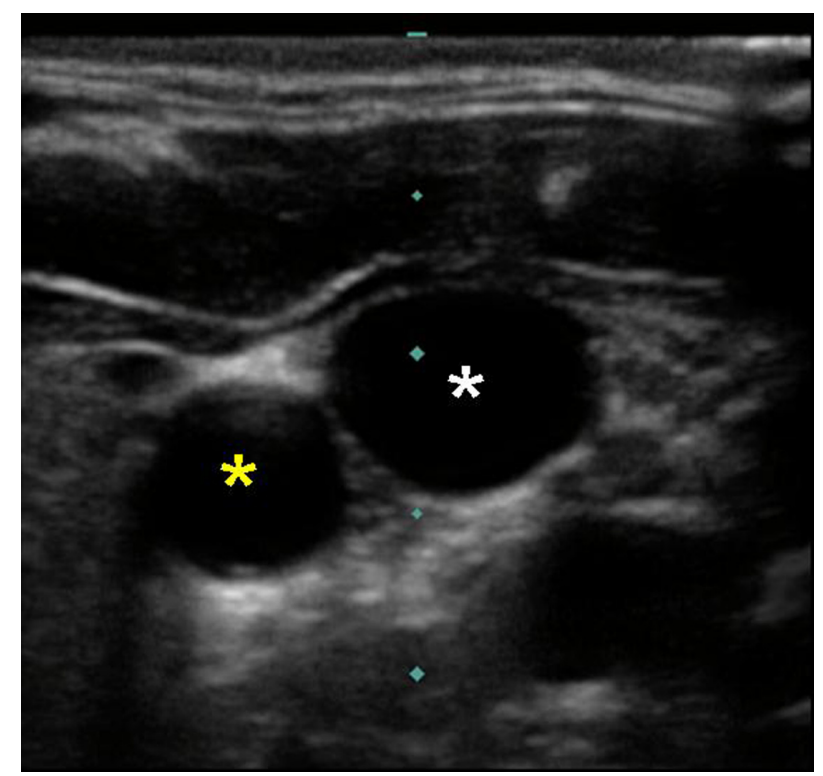

Figure 7 Ultrasound image of right carotid artery (yellow star) and right internal jugular vein (white star).

Common carotid artery diameter is reliably less than $1 \mathrm{~cm} \cdot{ }^{28-31}$ Internal jugular vein diameter is generally greater than one $1 \mathrm{~cm},{ }^{23,24,32}$ but may be smaller. ${ }^{21,33}$ Although the internal jugular vein may generally be differentiated from the common carotid artery by its larger diameter, the diameters of these vessels will rarely approximate each other, and therefore, the internal jugular vein and common carotid artery are most reliably differentiated by their compressibility and pulsation, respectively. The internal jugular vein is easily compressible; gentle pressure applied to the ultrasound probe will completely flatten the diameter of the internal jugular vein while leaving the diameter of the carotid artery unchanged (Figure 8). The cannulating needle and/or tissue compression it causes as it is advanced beneath the cutaneous surface can be visualized in real time by ultrasound, allowing the ultrasound image to guide puncture of the internal jugular vein at a 12 o'clock position and avoid unintended puncture of other structures.

Real-time ultrasound guidance has been shown to improve the technical efficiency and efficacy of internal jugular venous cannulation ${ }^{34-36}$ and to decrease the frequency of arterial punctures. ${ }^{35,36}$ Sonographic guidance has also decreased the frequency of hematoma, hemothorax, and pneumothorax. ${ }^{35}$

\section{Subclavian cannulation}

The subclavian vein, long favored by surgeons, ${ }^{37-39}$ offers an alternative to the internal jugular vein for central venous access. It may be associated with fewer infectious

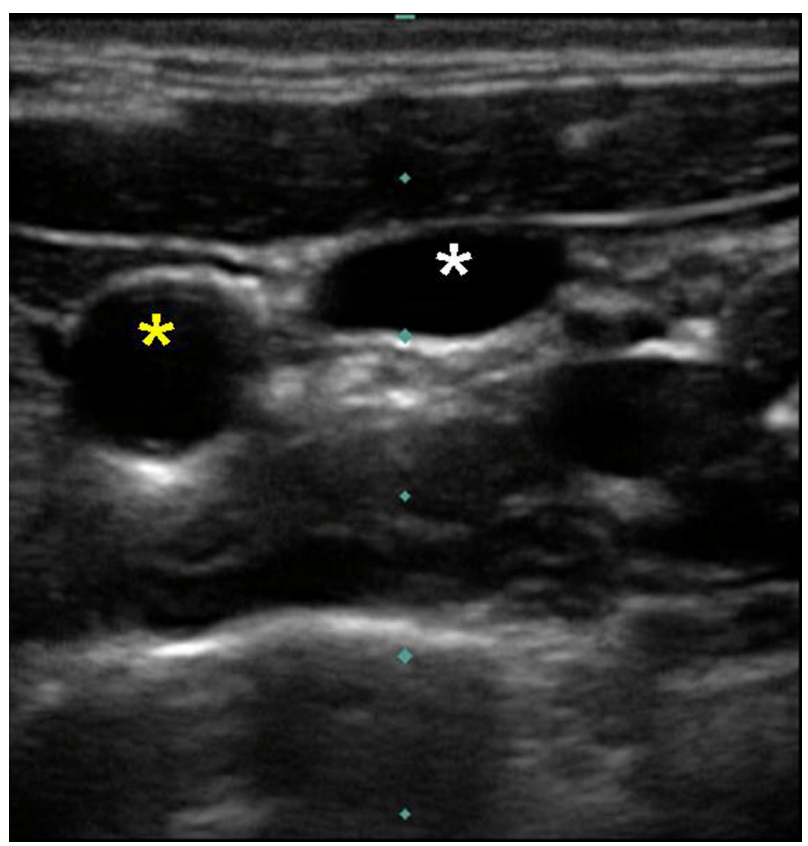

Figure 8 Ultrasound image of right internal jugular vein compressed by gentle probe pressure (white star) and carotid artery (yellow star).

complications than the internal jugular vein, ${ }^{1}$ and will remain accessible after localized thrombosis of the internal jugular vein. Well-designed studies comparing the internal jugular and subclavian approaches are sparse. Review of multiple nonrandomized studies suggests the arterial puncture is more frequent with the internal jugular approach, catheter malposition is more frequent with the subclavian approach, and the incidences of pneumothorax, hemothorax, and thrombosis are equivalent. ${ }^{40}$ The anatomic landmark approach to subclavian cannulation remains the standard of care and is our preferred and routine approach to this vessel. However, the enhanced safety of ultrasound in the internal jugular position increases interest in sonographically-assisted cannulation of the axillary vein as an alternative, ${ }^{41}$ but any advantage of this technique over landmark-guided subclavian cannulation remains unproven.

\section{Surface anatomy and venipuncture technique}

An infraclavicular approach to the subclavian vein is preferred by the authors. The operator stands on the side to be cannulated. A right-handed operator will find a position partially turned toward the patient's feet most comfortable for right-sided cannulation and a position turned partially toward the patient's head most comfortable for left-sided cannulation. The positions can be reversed for left-handed operators. The patient should be placed in the Trendelenburg 
position to maximize venous filling and minimize risk of air embolus; the head and neck position should be neutral.

The goal of subclavian venipuncture is to pass a needle inferior to the clavicle and superior to the first rib to access the subclavian vein as it courses over the first rib. The appropriate course for the needle passes immediately beneath the junction of the medial one-third and lateral two-thirds of the clavicle. This junction, ie, the "break" of the clavicle, ${ }^{42}$ is the point at which the anterior convexity of the medial clavicle transitions into an anterior concavity laterally (Figure 9). The appropriate point for cutaneous puncture lies 1-2 cm inferior and lateral to the clavicular transition point (Figure 9). Cutaneous puncture at this point facilitates passage of the needle inferior to the clavicle. A cutaneous puncture site closer to the clavicle creates difficulty maneuvering the needle beneath the clavicle. More medial cannulation may be impeded by calcification of the costoclavicular ligament. As the needle is advanced, it must remain absolutely parallel to the floor; if the needle is directed posteriorly to negotiate the clavicle, the risk of pneumothorax is greatly increased. Only gentle pressure from the operator's nondominant thumb is necessary to depress the needle in a flat coronal plane beneath the clavicle if the correct cutaneous puncture site is chosen. As the needle is advanced from the cutaneous puncture site to a point beneath the clavicular transition point, its tip should be aimed just above the tip of the operator's nondominant index finger placed in the sternal notch. The needle is advanced along this course passing through the subclavius muscle until the subclavian vein is accessed.

Kilbourne et al analyzed video recordings of surgical and emergency residents performing subclavian cannulation

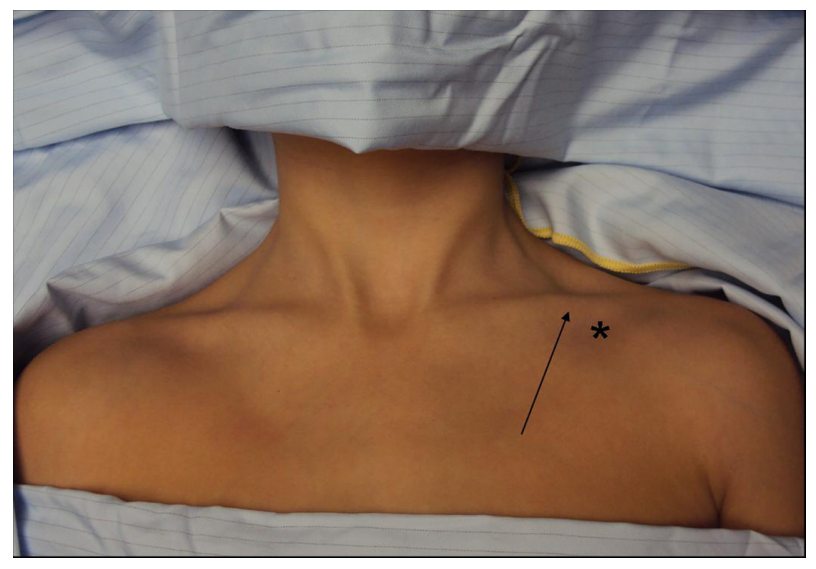

Figure 9 The "break" of the clavicle (arrow) and position of appropriate cutaneous puncture (black star). Note that head and neck drapes must always be placed with care to avoid potential airway obstruction and to allow appropriate airway assessment. during trauma resuscitation and identified six common technical errors. ${ }^{43}$ Five of these errors are directly related to anatomic considerations, and include cutaneous puncture too close to the clavicle, passage of the needle through the clavicular periosteum, too shallow a trajectory beneath the clavicle, failure to identify landmarks properly, and orientation of the needle in a cephalad direction away from the sternal notch. ${ }^{43}$ The final error is extravascular displacement of the needle after successful venipuncture but before guidewire placement. Kilbourne et al suggest that mentors' awareness of these errors can improve teaching effectiveness and enhance learners' safe practice. ${ }^{42}$

The authors use the infraclavicular approach with the clavicular break as the primary landmark as described above. Alternative landmarks may also be used to orient an infraclavicular approach to the subclavian vein. The deltoid tuberosity of the clavicle is a palpable anterior projection lying roughly at the junction of the lateral one-third and medial two-thirds of the clavicle. Using the deltoid tuberosity approach, the cutaneous puncture site is placed $1.5 \mathrm{~cm}$ inferior to the medial border of this landmark, and the needle is advanced toward the sternoclavicular joint and sternal notch. ${ }^{44}$ Other landmarks described include the coracoclavicular line ${ }^{45}$ and the deltopectoral triangle. ${ }^{46,39}$

The subclavian vein can also be cannulated using a supraclavicular approach. ${ }^{47}$ This route is preferred by some authors. ${ }^{48}$ The essential landmark for the supraclavicular approach is the junction of the lateral border of the clavicular head of the sternocleidomastoid with the clavicle. The point of cutaneous puncture lies $1 \mathrm{~cm}$ superior and $1 \mathrm{~cm}$ lateral to this junction. The junction of the sternocleidomastoid with the clavicle defines the claviculosternomastoid angle. The cannulating needle tip is angled posteriorly $5^{\circ}-15^{\circ}$ off a coronal plane and advanced along a line that bisects the claviculosternomastoid angle. This will lead to subclavian venipuncture between the clavicle and the anterior scalene muscle. ${ }^{47}$ Others suggest cutaneous puncture directly at the claviculosternomastoid angle and advancing the needle along the claviculosternomastoid angle bisector parallel and inferior to the clavicle to enter the vein at an insertion depth of $1-2 \mathrm{~cm} .{ }^{49}$ Anatomic data from three-dimensional computed tomography reconstructions suggest that, with the sternocleidomastoid-clavicular junction as a cutaneous puncture point, the needle should be oriented approximately $11^{\circ}$ medially and $35^{\circ}$ posteriorly as it is advanced approximately $1.4 \mathrm{~cm}$ to enter the vein; unfortunately relatively large standard deviations for these mean values limit their clinical utility. ${ }^{50}$ Supraclavicular 
subclavian cannulation can be facilitated by ultrasound guidance.

\section{Alternative positioning and adjunctive maneuvers}

Magnetic resonance images reveal that passive retraction of the shoulders by placement of a rolled towel between the shoulder blades leads to compression of the subclavian vein between the first rib and clavicle, ${ }^{51}$ which can be expected to impede successful cannulation. Contralateral head rotation increases the angle between the subclavian and internal jugular vein (normally at least $90^{\circ}$ ). This maneuver may thus increase the likelihood of passage of a catheter from the subclavian into the ipsilateral internal jugular vein rather than into the superior vena cava. ${ }^{51}$ Trendelenburg position does not increase the caliber of the subclavian vein which is held open in the flat, supine position by the fibrous attachments discussed below. ${ }^{51}$ The authors nonetheless recommend the Trendelenburg position when feasible to maximize venous filling and minimize risk of air embolus.

\section{Deep anatomic considerations Course}

As with the internal jugular veins, the deep courses of the right and left subclavian veins are not bilaterally symmetric. The venous course from the left subclavian vein arcs through the innominate vein to the superior vena cava in a gentle curve (Figure 2). However, the right subclavian vein makes a more sharply angled turn into the superior vena cava as it is joined by the internal jugular vein (Figure 3). This sharp angle of the right subclavian vein can lead to difficulties with placing catheters through introducer sheaths. If the introducer sheath lies too far medially relative to the lumen of the superior vena cava, lines may either kink as they pass into the superior vena cava or be passed into the contralateral subclavian and axillary veins. Related to the bilateral asymmetry, malpositioning of catheters into the contralateral subclavian or either internal jugular vein occurs more frequently from right than from left subclavian venous access. ${ }^{52}$

\section{Investing tissues}

The adventitia of the subclavian vein becomes invested with the connective tissue of the clavicular periosteum, the costoclavicular ligament, and the fascia of the subclavius and anterior scalene muscles. These soft tissue attachments suspend the vein and impede collapse even in instances of hypovolemia. ${ }^{42}$ Thus, cadaver dissections reveal the subclavian vein to be patulous even when the internal jugular and innominate veins are collapsed. ${ }^{51}$ This gives the subclavian approach a relative advantage over the internal jugular approach for patients in shock. A randomized study of patients with average systolic blood pressures of $80 \mathrm{mmHg}$ requiring acute volume resuscitation demonstrated that percutaneous subclavian cannulation and basilic vein cutdown were equally successful and were performed with equivalent complication rates, but percutaneous subclavian cannulation was performed six minutes sooner. ${ }^{53}$

\section{Pinch-off syndrome}

The close relationship between the clavicle and first rib may lead to crimping and intermittent positional or permanent occlusion of compressible catheters placed in this position. This complication has been referred to as the subclavian pinch-off syndrome..$^{54}$ This problem occurs with long-term venous access catheters which are more compressible than short-term catheters, and with an incidence of $1 \%-5 \% .55,56$ The resulting occlusion will render infusion and possibly aspiration through the line difficult or impossible. At times, infusion will depend upon the position of the patient's arm; occlusion occurs when the arm is at the patient's side and is relieved as the arm is raised. In a review of 112 reported cases, Mirza et al found the clinical presentation to be local pain and/or swelling in $60 \%$, and inability to flush or aspirate the catheter in $31 \% .{ }^{56}$ Infusion nurses will often be the first to recognize pinch-off symptoms and secure early diagnosis ${ }^{58}$ before catheter fracture and embolization occur. Luminal narrowing of the catheter under the clavicle is visible on chest $\mathrm{x}-\mathrm{ray}^{59}$ and can secure the diagnosis. This radiographic sign was termed the pinch-off sign. ${ }^{53}$ Indeed, Hinke et a ${ }^{54}$ has graded chest $\mathrm{x}$-ray findings, ie, $0=$ smooth curved subclavian course of catheter, 1 = any distortion of this course, $2=$ catheter narrowing, and $3=$ catheter transection and embolization. Grade 2 correlated with pinch-off syndrome, whereas Grade 1 did not. ${ }^{56}$ The consistency of demonstrating catheter luminal narrowing may be enhanced by chest $\mathrm{x}$-rays obtained with the patient's arms at his/her side. ${ }^{56}$

Aiken and Minton suggest that the occurrence of pinch- off syndrome is related to medial subclavian venipuncture and the triangular cross-sectional shape of the costoclavicular-scalene triangle ${ }^{53}$ formed by the medial clavicle anteriorly, the first rib inferiorly, and the anterior scalene muscle posteriorly. The more medially the catheter enters the vein, the more anteriorly it will lie within the acute angle formed between the clavicle and first rib. The more anteriorly the catheter lies, the more likely it will be compressed as the costoclavicular angle closes with an upright position. 
With a relatively lateral venipuncture, the catheter will pass through the wider, posterior portion of this triangular space (and with a likely intravenous course) rendering compression with arm abduction less likely than if the catheter lies in a more anterior position.

Although rare, the pinch-off syndrome demands immediate attention. Over time, repeated crimping of the catheter will weaken its mechanical integrity, eventuating in fracture and catheter embolus. The incidence of transection and embolization has been estimated to be $1.6 \% 0^{57}$ of percutaneously placed subclavian catheters. The incidence of embolization in pinch-off syndrome is reported as high as $80 \%,{ }^{57}$ but this likely reflects publication bias. The reported mortality of embolization is $1.8 \%{ }^{60}$ and emphasizes the need for both awareness of pinch-off syndrome and proper evaluation of malfunctioning catheters. Indeed, it seems insightful nursing assessments may diminish the incidence of embolus to $20 \%$ of patients with pinch-off syndrome. ${ }^{61}$ Overall, fragment embolization is very rare and occurs in $0.12 \%$ of patients with silicone catheters. Catheter embolus may be asymptomatic in $24 \%$ of cases, but most commonly (56\%) presents as catheter malfunction; less frequent symptoms include arrhythmia, pulmonary complaints, and sepsis. ${ }^{60}$ There are other causes of catheter embolization, but pinch-off syndrome is responsible for $41 \%$ of cases. ${ }^{60}$ For this reason, pinch-off syndrome should be treated with catheter removal ${ }^{57}$ and insertion of a catheter in a new site. In the vast majority of cases, embolized fragments can be removed with percutaneous transvenous techniques; however, fragments have been found adherent to vascular walls in $4.2 \%$ of cases and have required open thoracotomy in $2.3 \% .{ }^{60}$ Some suggest subclavian pinch-off syndrome can be avoided by puncture of the subclavian vein at a relatively lateral position.

\section{Anatomic complications}

Beginning at the axillary-subclavian junction, the posterior relationships of the subclavian vein are first the anterior scalene muscle with the phrenic nerve lying on its anterior face, followed by the subclavian artery, and then the brachial plexus. The risk of subclavian arterial puncture increases as the probing needle is aimed progressively posteriorly. Although the anterior scalene muscle, $1-1.5 \mathrm{~cm}$ thick, provides relative protection of the subclavian artery during attempted subclavian venipuncture at this level, ${ }^{37}$ rotating the needle tract in an increasingly posterior direction away from the sternal notch will create progressively shorter traverses to the subclavian artery and increase risk of arterial puncture.
Subclavian artery puncture is avoided by aiming the venipuncture needle just superior to the tip on the operator's index finger placed in the sternal notch. Lateral to the first rib, the axillary artery and nerves lie directly posterior to the axillary vein without an interposed muscle; arterial puncture is thus even more likely with lateral cutaneous punctures ${ }^{37}$ if the needle tract is oriented posteriorly. Although direct brachial plexus injury is unlikely, hematoma resulting from arterial puncture may cause a compression neuropathy.

Medial to the anterior scalene muscle and first rib, the posterior relationships of the subclavian vein are the phrenic nerve (as it swings medially off the muscle), the internal mammary artery, and the apical pleura. The subclavian artery arcs more superiorly here so the vein is primarily apposed to the pleura. Thus, a venipuncture needle advanced too deeply at this level will puncture the pleura and lung. Given the conical shape of the superior chest cavity, inferior (caudad) angulation of the probing needle places the needle on a shorter course to the pleura (either through or inferior to the vein) and increases the likelihood of pneumothorax. Advancing the needle perfectly horizontally in a coronal plane places the needle on a trajectory that maximizes an encounter with vein before pleura. Consequently, pneumothorax is avoided by keeping the needle and syringe absolutely parallel to the floor. If the operator lifts the needle and syringe off the chest wall, angling the needle tip more deeply, passage into the pleural space and lung becomes much more likely because the subclavian veins are immediately apposed to pleura posteriorly (Figures 10 and 11). For this reason, chronic forward rotation of the shoulders which prevent a flat horizontal course for the cannulating needle is a relative contraindication to subclavian cannulation.

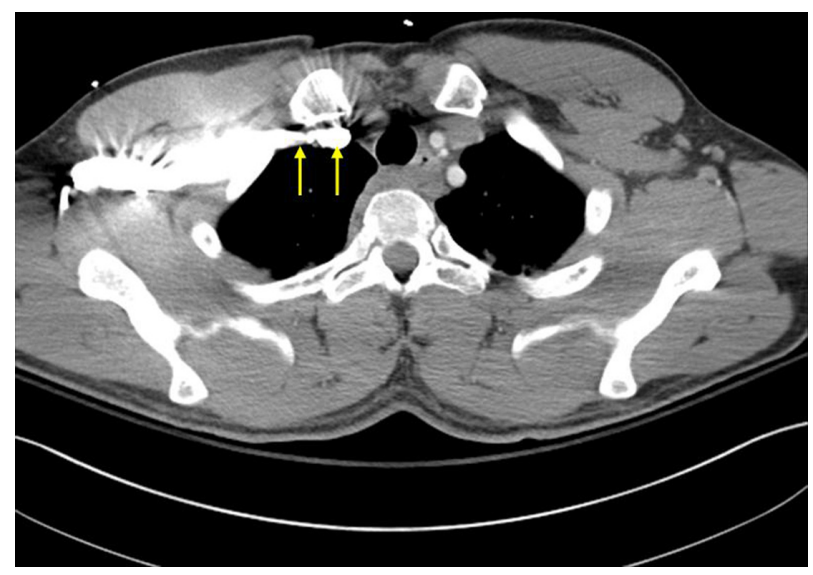

Figure 10 Computed tomography scan demonstrating apposition of right subclavian vein to pleura (arrows). 


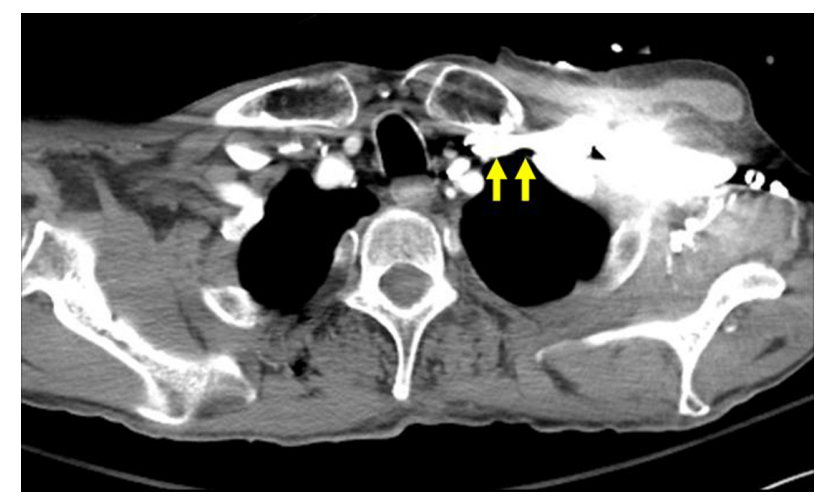

Figure I I Computed tomography scan demonstrating apposition of left subclavian vein to pleura (arrows).

\section{Role of ultrasound}

The authors do not use ultrasound for subclavian catheterization. Ultrasound visualization of the subclavian vein can confirm patency or identify thrombosis. However, the anatomic position of the subclavian vein between the clavicle and first rib complicates real-time ultrasound localization during venipuncture. ${ }^{1}$ Ultrasound localization without real-time ultrasound guidance does not improve success of subclavian venipuncture attempts, nor does it decrease complication rates. ${ }^{62}$ Real-time ultrasound localization of the subclavian vein through the window between the clavicle and first rib is difficult and may generate unsatisfactory images ${ }^{41}$ However, the axillary vein and artery can be easily visualized with ultrasound more laterally on the chest wall anterior to the lateral clavicle. Here, the axillary vein can be recognized as a compressible structure lying anterior to the noncompressible, pulsating axillary artery. Ultrasoundguided cannulation of the axillary vein may prove to be a safer technique than blind subclavian cannulation, ${ }^{63,64}$ especially for those operators without extensive experience with the latter approach. ${ }^{65}$ Blind approaches to the axillary vein have been described, ${ }^{66}$ but there is no reason to pursue these if ultrasound is available.

\section{Femoral cannulation}

Femoral cannulation may be favored during emergent resuscitation when procedures such as endotracheal intubation and cardiac compressions limit safe concurrent access to the internal jugular vein and subclavian vein.

\section{Surface anatomy and venipuncture technique}

Femoral venipuncture is performed in the anterior superior thigh, just below the level of the inguinal ligament. This ligament runs from the pubic tubercle medially to the anterior superior iliac spine laterally. The femoral artery generally bisects the inguinal ligament. The femoral vein lies immediately medial to the femoral artery. Cutaneous puncture is performed approximately $1 \mathrm{~cm}$ medial to the point of maximal pulsation of the femoral artery. As with venipuncture of the internal jugular and axillary veins, venipuncture of the femoral vein can be facilitated by realtime ultrasound localization.

It is critical to ensure that the puncture of the vein occurs below the level of the inguinal ligament, ie, meaning the skin insertion point should be slightly inferior on the thigh. Puncture above the inguinal ligament is in fact a puncture of the external iliac vein which quickly becomes a deep retroperitoneal structure making it difficult to place pressure on the insertion site if bleeding occurs.

\section{Deep anatomic considerations}

The deep courses of the femoral veins are bilaterally symmetric. Both veins gently arc medially to the inguinal ligament, becoming the external iliacs and coursing further medially to the inferior vena cava. Each femoral vein provides equally efficacious access and can be employed in the correct setting as defined below.

\section{Anatomic complications}

Femoral vein catheters are associated with higher rates of infection and thrombosis than subclavian catheters or internal jugular vein catheters. ${ }^{67}$ Therefore, the femoral vein is considered the third choice for catheterization and is used only when subclavian and internal jugular approaches are not feasible. For example, femoral cannulation becomes a reasonable option in patients with extensive upper extremity trauma and/or thrombosis. Also, during cardiopulmonary resuscitation, the femoral vein can be cannulated while cardiopulmonary resuscitation is in progress. The caveat to this is that the femoral vein may be collapsed in such a patient, making cannulation quite challenging.

\section{Role of ultrasound}

Ultrasound may be a useful adjunct in the placement of femoral catheters. Much like the technique for internal jugular cannulations, the ultrasound probe can be placed in a sterile sheath and be used in real time to visualize the puncture. The femoral vein will be a typically larger caliber, compressible structure anteromedial to the femoral vein which should be noncompressible and pulsatile. 


\section{Conclusion}

Venous cannulation has been revolutionized with the advent of percutaneous approaches. The use of surface anatomical landmarks is critical to the success of these procedures. While use of ultrasound localization adds a dimension of orientation, the surface landmarks are still an instrumental component of safe central venous cannulation. Thorough understanding of these anatomical landmarks, including their relationships to the underlying structures, is critical to minimize complications and optimize success rates.

\section{Disclosure}

The authors report no conflicts of interest in this work.

\section{References}

1. McGee DC, Gould MK. Preventing complications of central venous catheterization. $N$ Engl J Med. 2003;348:1123-1133.

2. Ellis H. The clinical anatomy of the great veins of the neck. Br J Hosp Med. 2010;71(2):M20-M21.

3. Boon JM, Van Schoor AN, Abrahams PH, Meiring JH, Welch T, Shanahan D. Central venous catheterization - An anatomical review of a clinical skill - Part 1: Subclavian vein via the infraclavicular approach. Clin Anat. 2007;20:602-611.

4. Boon JM, Van Schoor AN, Abrahams PH, Meiring JH, Welch T. Central venous catheterization - An anatomical review of a clinic skill, Part 2: Internal jugular vein via the supraclavicular approach. Clin Anat. 2008; 21:15-22.

5. Maki DG, Ringer M, Alvarado CJ. Prospective randomized trial of povidone-iodine, alcohol, and chlorhexidine for prevention of infection associated with central venous and arterial catheters. Lancet. 1991; 338:339-343.

6. Mimoz O, Pieroni L, Lawrence C, et al. Prospective, randomized trial of two antiseptic solutions for prevention of central venous or arterial catheter colonization and infection in intensive care unit patients. Crit Care Med. 1996;24:1818-1823.

7. Raad II, Hohn DC, Gilbreath BJ, et al. Prevention of central venous catheter-related infections by using maximal sterile barrier precautions during insertion. Infect Control Hosp Epidemiol. 1994;15:231-238.

8. American Association of Clinical Anatomists, Educational Affairs Committee. The clinical anatomy of several invasive procedures. Clin Anat. 1999;12:43-54.

9. Seldinger SI. Catheter replacement of the needle in percutaneous Arteriography: A new technique. Acta Radiol. 1953;39:368-376.

10. Pikwer A, Baath L, Davidson B, Perstoft I, Akeson J. The incidence and risk of central venous catheter malpositioning: A prospective cohort study in 1619 patients. Anaesth Intensive Care. 2008;36:30-37.

11. Aslamy Z, Dewald CL, Heffner JE. MRI of central venous anatomy: Implications for central venous catheter insertion. Chest. 1998; 114:820-826.

12. Andrews RT, Bova DA, Venbrux AC. How much guidewire is too much? Direct measurement of the distance from subclavian and internal jugular vein access sites to the superior vena cava-atrial junction during central venous catheter placement. Crit Care Med. 2000;28:138-142.

13. Collier PE, Goodman GB. Cardiac tamponade caused by central venous catheter perforation of the heart: A preventable complication. J Am Coll Surg. 1995;181:460-463.

14. National Institute for Clinical Excellence. Guidance on the use of ultrasound locating devices for placing central venous catheters. London: National Institute for Clinical Excellence; 2002.
15. Milling TJ Jr, Rose J, Briggs WM, et al. Randomized, controlled clinical trial of point-of-care limited ultrasonography assistance of central venous cannulation: The Third Sonography Outcomes Assessment Program (SOAP-3) Trial. Crit Care Med. 2005;33:1764-1769.

16. Blaivas M, Adhikari S. An unseen danger: Frequency of posterior vessel wall penetration by needles during attempts to place internal jugular vein central catheters using ultrasound guidance. Crit Care Med. 2009;37:2345-2349.

17. Cahill DR. Central venous catheterization. In: Lachman's Case Studies in Anatomy. 4th ed. New York, NY: Oxford University Press; 1997.

18. Chudhari LS, Karmarkar US, Dixit RT, Sonia K. Comparison of two different approaches for internal jugular vein cannulation in surgical patients. J Postgrad Med. 1998;44:57-62.

19. Sulek CA, Blas ML, Lobato EB. A randomized study of left versus right internal jugular vein cannulation in adults. J Clin Anesth. 2000; 12:142-145.

20. Chandrasekaran S, Chandrasekaran VP. Anatomical variations of the internal jugular vein in relation to common carotid artery in lesser supra clavicular fossa - a colour doppler study. Int J Basic Med Sci. 2010;1(4). Available at: http://www.ijbms.com/anatomy/supra-clavicularfossa- $\%$ e2\%80\%93-a-colour-doppler-study/. Accessed December 15, 2010.

21. Turba UC, Uflacker R, Hannegan C, Selby JB. Anatomic relationship of the internal jugular vein and the common carotid artery applied to percutaneous transjugular procedures. Cardiovasc Intervent Radiol. 2005;28:303-306.

22. Khatri VP, Wagner-Sevy S, Espinosa MH, Fisher JB. The internal jugular vein maintains its regional anatomy and patency after carotid endarterectomy: A prospective study. Ann Surg. 2001;233:282-286.

23. Bazaral M, Harlan S. Ultrasonographic anatomy of the internal jugular vein relevant to percutaneous cannulation. Crit Care Med. 1981;9: 307-310.

24. Sulek CA, Gravenstein N, Blackshear RH, Weiss L. Head rotation during internal jugular vein cannulation and the risk of carotid artery puncture. Anesth Analg. 1996;82:125-128.

25. Suarez T, Baerwald JP, Kraus C. Central venous access: The effects of approach, position, and head rotation on internal jugular vein cross-sectional area. Anesth Analg. 2002;95:1519-1524.

26. Marcus HE, Bonkat E, Dagtekin O, et al. The impact of Trendelenburg position and positive end-expiratory pressure on the internal jugular cross-sectional area. Anesth Analg. 2010;111:432-436.

27. Arndt JO, Klauske J, Mersch F. The diameter of the intact carotid artery in man and its change with pulse pressure. Pflugers Arch Gesamte Physiol Menschen Tiere. 1968:301:230-240.

28. Eigenbrodt ML, Bursac Z, Rose KM, et al. Common carotid arterial interadventitial distance (diameter) as an indicator of the damaging effects of age and atherosclerosis, a cross-sectional study of the atherosclerosis risk in community cohort limited access data (ARICLAD), 19871989. Cardiovasc Ultrasound. 2006;4(1):1476-1486. Available at: http://www.cardiovascularultrasound.com/content/4/1/1. Accessed November 9, 2010.

29. Olson RM. Human carotid artery wall thickness, diameter, and blood flow by a noninvasive technique. J Appl Physiol. 1974;37:955-960.

30. Studinger P, Lénárd Z, Kováts Z, Kocsis L, Kollai M. Static and dynamic changes in carotid artery diameter in humans during and after strenuous exercise. J Physiol. 2003;550:575-583.

31. Dhulkhed V, Reddy A, Gupta A, Dhulkhed P. An observational study of change in diameter of right internal jugular vein with various body positions in volunteers with the aid of 2-dimensional ultrasonography. Internet J Anaesth. 2009;21(2). Available at: http://www.ISPUB.com. Accessed December 16, 2010.

32. Muhammad JK, Pugh ND, Boden L, Crean St J, Fardy MJ. The effect of head rotation on the diameter of the internal jugular vein: Implications for free tissue transfer. J Craniomaxillofac Surg. 2001;29:214-218.

33. Hind D, Calvert N, McWilliams R, et al. Ultrasonic locating devices for central venous cannulation: Meta-analysis. BMJ. 2003;327:361-364. 
34. Karakitsos D, Labropoulos N, De Groot E, et al. Real-time ultrasoundguided catheterisation of the internal jugular vein: A prospective comparison with the landmark technique in critical care patients. Crit Care. 2006;10:R162. Available at: http://ccforum.com/content/10/6/R162. Accessed November 1, 2010.

35. Troianos CA, Jobes DR, Ellison N. Ultrasound-guided cannulation of the internal jugular vein. A prospective, randomized study. Anesth Analg. 1991;72:823-826.

36. Moosman DA. The anatomy of infraclavicular subclavian vein catheterization and its complications. Surg Gynecol Obstet. 1973;136: 71-74.

37. Linos DA, Mucha P, van Heerden JA. Subclavian vein: A golden route. Mayo Clin Proc. 1980;55:315-321.

38. Thompson EC, Calver LE. How I do it: Safe subclavian vein cannulation. Am Surg. 2005;71:180-183.

39. Ruesch S, Walder B, Tramer MR. Complications of central venous catheters: Internal jugular versus subclavian access - A systematic review. Crit Care Med. 2002;30:454-460.

40. Sandhu NS. Transpectoral ultrasound-guided catheterization of the axillary vein: An alternative to standard catheterization of the subclavian vein. Anesth Analg. 2004;99:183-187.

41. Cahill DR. Central venous catheterization. In: Lachman's Case Studies in Anatomy. 4th ed. New York, NY: Oxford University Press; 1997.

42. Kilbourne MJ, Bochicchio GV, Scalea T, Xiao Y. Avoiding common technical errors in subclavian central venous catheter placement. J Am Coll Surg. 2009;208:104-109.

43. Von Goedecke A, Keller C, Moriggl B, et al. An anatomic landmark to simplify subclavian vein cannulation: The "deltoid tuberosity". Anesth Analg. 2005;100:623-628.

44. Tripathi M, Tripathi M. Subclavian vein cannulation: An approach with definite landmarks. Ann Thorac Surg. 1996;61:238-240.

45. Moran SG, Peoples JB. The deltopectoral triangle as a landmark for percutaneous infraclavicular cannulation of the subclavian vein Angiology. 1993;44:683-686.

46. Czarnik T, Gawda R, Perkowski T, Weron R. Supraclavicular approach is an easy and safe method of subclavian vein catheterization even in mechanically ventilated patients. Anesthesiology. 2009;111:334-339.

47. Cunningham SC, Gallmeier E. Supraclavicular approach for centra venous catheterization: "Safer, simpler, speedier". J Am Coll Surg. 2007;205:514-516.

48. Nevarre DR, Domingo OH. Supraclavicular approach to subclavian catheterization: review of the literature and results of 178 attempts by the same operator. J Trauma. 1997;42:305-309.

49. Jung CW, Seo JH, Lee W, Bahk JH. A novel supraclavicular approach to the right subclavian vein based on three-dimensional computed tomography. Anesth Analg. 2007;105:200-204.

50. Jesseph JM, Conces DJ, Augustyn GT. Patient positioning for subclavian vein catheterization. Arch Surg. 1987;122:1207-1209.
51. Unal AE, Bayar S, Arat M, Ilhan O. Malpositioning of Hickman catheters, left versus right sided attempts. Transfus Apher Sci. 2003; 28:9-12.

52. Arrighi DA, Farnell MB, Mucha P, Ilstrup DM, Anderson DL. Prospective, randomized trial of rapid venous access for patients in hypovolemic shock. Ann Emerg Med. 1989;18:927-930.

53. Aitken DR, Minton JP. The "pinch-off sign": A warning of impending problems with permanent subclavian catheters. Am J Surg. 1984;148: 633-636.

54. Hinke DH, Zandt-Stastny DA, Goodman LR, Quebbeman EJ, Krzywda EA, Andris DA. Pinch-off syndrome: A complication of implantable subclavian venous access devices. Radiology. 1990;177: 353-356.

55. Koonings PP, Given FT. Long-term experience with a totally implanted catheter system in gynecologic oncologic patients. J Am Coll Surg. 1994;178:164-166.

56. Mirza B, Vanek VW, Kupensky DT. Pinch-off syndrome: Case report and collective review of the literature. Am Surg. 2004;70:635-644.

57. Andris DA, Krzywda EA. Catheter pinch-off syndrome: Recognition and management. J Intraven Nurs. 1997;20:233-237.

58. Lafreniere R. Indwelling subclavian catheters and a visit with the "pinched-off sign". J Surg Oncol. 1991;47:261-264.

59. Surov A, Wienke A, Carter JM, et al. Intravascular embolization of venous catheter - causes, clinical signs, and management: A systematic review. J Parenter Enteral Nutr. 2009;33:677-685.

60. Andris DA, Krzywda EA, Schulte W, Ausman R, Quebbeman EJ. Pinch-off syndrome: A rare etiology for central venous catheter occlusion. J Parenter Enteral Nutr. 1994;18:531-533.

61. Mansfield PF, Hohn DC, Fornage BD, Gregurich MA, Ota DM. Complications and failures of subclavian-vein catheterization. $N$ Engl J Med. 1994;331:1735-1738.

62. Pirotte T. Ultrasound-guided vascular access in adults and children: Beyond the internal jugular vein puncture. Acta Anaesthesiol Belg. 2008;59:157-166.

63. Skolnick ML. The role of sonography in the placement and management of jugular and subclavian central venous catheters. AJR Am J Roentgenol. 1994;163:291-295.

64. Gualtieri E, Deppe SC, Sipperly ME, Thompson DR. Subclavian venous catheterization: Greater success rate for less experienced operators using ultrasound guidance. Crit Care Med. 1995;23:692-697.

65. Taylor BL, Yellowlees I. Central venous cannulation using the infraclavicular axillary vein. Anesthesiology. 1990;72:55-58.

66. Merrer J, De Jonghe B, Golliot F, et al. Complications of femoral and subclavian venous catheterization in critically ill patients. JAMA. 2001; 286:700-707.
Risk Management and Healthcare Policy

\section{Publish your work in this journal}

Risk Management and Healthcare Policy is an international, peerreviewed, open access journal focusing on all aspects of public health, policy, and preventative measures to promote good health and improve morbidity and mortality in the population. The journal welcomes submitted papers covering original research, basic science, clinical \& epidemio-

\section{Dovepress}

logical studies, reviews and evaluations, guidelines, expert opinion and commentary, case reports and extended reports. The manuscript management system is completely online and includes a very quick and fair peerreview system, which is all easy to use. Visit http://www.dovepress.com/ testimonials.php to read real quotes from published authors. 